Article

\title{
Effect of the Sulfonation on the Swollen State Morphology of Styrenic Cross-Linked Polymers
}

\author{
Chiara Dalla Valle, Marco Zecca, Federico Rastrelli $@$, Cristina Tubaro $@$ and Paolo Centomo *(i) \\ Department of Chemical Sciences, University of Padova, Via Marzolo 1, 35131 Padova, Italy; \\ chiara.dallavalle@studenti.unipd.it (C.D.V.); marco.zecca@unipd.it (M.Z.); federico.rastrelli@unipd.it (F.R.); \\ cristina.tubaro@unipd.it (C.T.) \\ * Correspondence: paolo.centomo@unipd.it; Tel.: +39-049-827-5206
}

Received: 23 December 2019; Accepted: 23 February 2020; Published: 6 March 2020

\begin{abstract}
The chemical structure and morphology of a set of sulfonic gel-type poly(styrene-divinylbenzene) resins ( $2 \mathrm{~mol} \% \mathrm{DVB}$ ) prepared with different synthetic approaches were investigated by solid state NMR, Inverse Size Exclusion Chromatography (ISEC), FT-IR and elemental analysis to compare their swollen state structure. FT-IR and solid state NMR clearly show that the sulfonation mainly occurs in the para- position with respect the main polymer chain. Sensible proportions of sulfone bridges were found in the materials obtained with oleum and chlorosulfonic acid. With oleum, the presence of the sulfone bridges is clearly associated to a reduced ability to swell in the water medium relative to the proton exchange capacity. This highlights the cross-linking action of the sulfone bridges according to ISEC results, showing a high proportion of a dense polymer fraction in the swollen material. An even higher degree of sulfone-bridging, lower swelling ability, and a high proportion of a dense polymer fraction in the swollen material are found in the resin obtained with chlorosulfonic acid. As a matter of fact, Cross Polarization Magic Angle Spinning Nuclear Magnetic Resonance (CP-MAS $\left.{ }^{13} \mathrm{C}-\mathrm{NMR}\right)$, elemental analysis, and ion exchange capacity, show that oleum and chlorosulfonic acid produced resins with remarkably smaller pores and lower swollen gel volume in polar solvents, with respect to concentrated sulfuric acid.
\end{abstract}

Keywords: swollen state porosity; crosslinked polymers; sulfone bridges; ISEC; CP-MAS ${ }^{13}$ C-NMR

\section{Introduction}

Sulfonic resins based on polystyrene-divinylbenzene (PS-DVB) are widely used in the fields of acid catalysis, ion exchange, and separation processes [1,2]. They must be used in the presence of a suitable liquid phase (water, an aqueous solution, or a polar organic solvent) able to swell the polymer framework: under these conditions an extensive nanometer size porosity with a surface area of hundreds of square meters per gram is generated [3-5]. The investigation of the morphological features of this porous system, representing the actual working arena of the resin requires suitable solution state techniques, such as Inverse Size Exclusion Chromatography (ISEC) [3,5-7], Electron Paramagnetic Resonance (EPR) investigation of the rotational motion of suitable nitroxide probes $[8,9]$, and time domain NMR spectroscopy [10], to rationalize the physico-chemical behaviour of these materials [11-13].

The direct sulfonation of PS-DVB resins with either sulfuric acid or oleum, or with chlorosulfonic acid and subsequent alkaline hydrolysis [14], introduces not only the sulfonic groups, but also sulfone bridges between pairs of phenyl rings. These bridges not only reduce the effective degree of sulfonation, but also represent a form of cross-linking which adventitiously adds to the cross-links produced by DVB. The elucidation of post cross-linking of PS-DVB resins at the molecular level has been addressed so far only in an early report by Goldstein and co-workers [15] and in a more recent 
one by Sherrington [16]. However, they were both devoted to the formation of cross-links upon Friedel-Crafts reactions catalyzed by Lewis acids such as $\mathrm{FeCl}_{3}$ and $\mathrm{ZnCl}_{2}$. In one case [16], this was the unpredicted result of the chloromethylation of PS-DVB with (chloromethyl)methylether during the production of anion-exchange resins. Solid state ${ }^{13} \mathrm{C}-\mathrm{NMR}$ allowed to characterize the parent resin, all the intermediate products and the final ion-exchanger. The degree of post cross-linking (methylene bridges between phenyl ring pairs) was quantified in $50-60 \%$. As solid state ${ }^{13} \mathrm{C}-\mathrm{NMR}$ still represents a state-of-the-art technique for the characterization of polymers [17] in the solid state, we decided to take a similar approach to address the problem of sulfone bridges formation during PS-DVB sulfonation with different agents. In this way, a semi-quantitative evaluation of the content of sulfone bridges was achieved. Moreover, it is well known that changes in the cross-linking degree of resins affects their swelling behaviour (as a rule of thumb, the higher the former, the smaller the latter). Therefore, we also investigated on the effect of the additional sulfone-bridge cross-links on the swollen state morphology of the ion-exchangers. For this purpose, the swollen sulfonated resins were characterized by ISEC in order to disclose differences in the porosity of ion-exchangers prepared with different sulfonating agents. All these pieces of information might be useful for better controlling the production of sulfonated ion-exchange resins and tailoring their morphology to specific practical applications under solid-liquid condition processes.

\section{Materials and Methods}

The starting material for the sulfonation is a gel-type styrene-co-divinylbenzene polymer with a nominal DVB content of $2 \%$ mol which was provided by Spolchemie, Usti nad Labem, Czech Republic. Reagents and solvents were purchased from Sigma-Aldrich and used as received.

\subsection{Sulfonation with Concentrated Sulfuric Acid}

Ca. $2 \mathrm{~g}$ of polymer were allowed to swell overnight in the least volume of 1,2-dichloroethane (DCE) as the solvent $(10-20 \mathrm{~mL})$, in a jacketed glass reactor equipped with a back condenser. $50-100 \mathrm{~mL}$ of $98 \% \mathrm{H}_{2} \mathrm{SO}_{4}$ (acid:solvent, 5:1 $\mathrm{v} / \mathrm{v}$ ) were slowly added to the swollen polymer. The mixture was heated up to $80^{\circ} \mathrm{C}$ for $3 \mathrm{~h}$, under moderate magnetic stirring and then cooled to room temperature. The reaction mixture was diluted with five portions of ca. $30 \mathrm{~mL}$ of sulfuric acid with a progressively lower concentration ( $10 \mathrm{M} ; 5 \mathrm{M} ; 2.5 \mathrm{M} ; 1 \mathrm{M} ; 0.1 \mathrm{M}$ ); a flow of cold water through the jacket of the reactor was used to prevent overheating. Finally, the resin was recovered by filtration, washed with distilled water up to neutral $\mathrm{pH}$ and dried at $110^{\circ} \mathrm{C}$.

\subsection{Sulfonation with Oleum}

The procedure is the same used for the sulfonation with sulfuric acid, but oleum replaced sulfuric acid as the sulfonating agent.

\subsection{Sulfonation with Chlorosulfonic Acid}

The resin (ca. $2 \mathrm{~g}$ ) was pre-swollen overnight with 1,2-dichloroethane in a glass jacketed reactor. $24 \mathrm{~mL}$ of 1,2-dichloroethane and $16 \mathrm{~mL}$ of $\mathrm{HClSO}_{3}$ were added under magnetic stirring and the mixture was let to react for three hours at $42{ }^{\circ} \mathrm{C}$. Subsequently, $50 \mathrm{~mL}$ of cold distilled water was added dropwise to the reaction mixture, maintained at low temperature with an ice bath. The chlorosulfonic resin was then recovered by vacuum filtration (G3 gooch), washed with distilled water until neutral $\mathrm{pH}$ and suspended in $40 \mathrm{~mL}$ of a $2 \%$ wt solution of $\mathrm{NaOH}$ in 1,4-dioxane:water $1: 1 \mathrm{v} / \mathrm{v}$, at $40^{\circ} \mathrm{C}$ for two hours. The resin was again recovered by vacuum filtration (gooch G3) and washed with a $10 \% \mathrm{HCl}$ solution in 1,4-dioxane:water 1:1 $v / v$, up to the complete removal of the chloride ions from the filtrate $\left(\mathrm{AgNO}_{3}\right.$ test). The solid was finally washed with $50 \mathrm{~mL}$ methanol and dried at $110^{\circ} \mathrm{C}$ overnight. 


\subsection{Determination of the Proton-Exchange Capacity of Sulfonic Resins}

Proton exchange capacity (PEC) is determined by acid-base back titration. About $100 \mathrm{mg}$ of resin was exactly weighted after drying overnight at $110^{\circ} \mathrm{C}$ in an oven. Each sample was treated with $10.0 \mathrm{~mL}$ of a standard aqueous solution (ca. $0.1 \mathrm{M}$ ) of $\mathrm{NaOH}$ in a $50 \mathrm{~cm}^{3}$ stoppered Erlenmeyer flask. The suspension was mechanically swirled (orbiting plate) overnight. To take into account the possible reaction with atmospheric $\mathrm{CO}_{2}$, a blank with $10.0 \mathrm{~mL}$ of the $\mathrm{NaOH}$ solution was prepared as well. The solutions (sample and blank) were eventually titrated with standard $0.1 \mathrm{M} \mathrm{HCl}$ and phenolphthalein as the indicator. The apparent specific content of sulfonic groups was calculated as the difference of the initial and final millimoles of $\mathrm{NaOH}$ (corrected for over-consumption due to $\mathrm{CO}_{2}$, if any) divided by the resin mass.

\subsection{ISEC Investigation}

The resin was loaded as the stationary phase in a void HPLC column and the elution volumes of standard solutes with known molecular size are measured in a HPLC apparatus modified ad-hoc [6]. The measurements were performed using a $0.2 \mathrm{M}$ sodium sulfate solution as the mobile phase and $\mathrm{D}_{2} \mathrm{O}$, sugars and dextrans as standard solutes. Further details can be found in the Supplementary Information and, for an extensive description of the technique, in ref. [3].

\subsection{Solid State NMR}

NMR spectra in the solid state were collected on a Varian 400 equipped with a narrow bore, triple resonance T3 Magic Angle Spin (MAS) probe spinning $4 \mathrm{~mm}$ rotors and operating at ${ }^{1} \mathrm{H}$ and ${ }^{13} \mathrm{C}$ frequencies of 400.36 and $100.68 \mathrm{MHz}$, respectively. The nominal temperature of the probe was always set to 298 K. Cross Polarization Magic Angle Spinning Nuclear Magnetic Resonance (CP-MAS ${ }^{13} \mathrm{C}$ NMR) spectra were acquired at $10 \mathrm{kHz}$ MAS with $1200 \mathrm{scans}$ and a repetition delay of $3 \mathrm{~s}$. The contact time for $\mathrm{CP}$ was $1 \mathrm{~ms}$, and an acquisition time of $50 \mathrm{~ms}$ was used. The chemical shifts were referenced against the $\mathrm{CH}_{2}$ resonance observed for adamantane at $38.48 \mathrm{ppm}$ with respect to the signal for neat tetramethylsilane (TMS). In order to homogenize the samples, resin beads were ground to obtain a fine powder prior to insertion into the rotor.

\section{Results and Discussion}

\subsection{Proton Exchange Capacity (PEC)}

The comparison of the PECs of the ion-exchange resins obtained with different sulfonation methods with the respective sulfur contents from the elemental analysis can give valuable information. The PECs of the polymer materials determined by back-titration are reported in Table 1.

Table 1. Analytical data on the composition of the sulfonic resins.

\begin{tabular}{cccccc}
\hline Sample $^{\mathbf{1}}$ & PEC $^{\mathbf{2}}$ & $\mathbf{C} \% \mathbf{w t}$ & Total S\% wt & $\mathbf{C l} \% \mathbf{w t}$ & $\mathbf{x}^{\mathbf{3}}$ \\
\hline Gel_oleum & 5.51 & 44.96 & $19.99(6.24)^{2}$ & - & 1.33 \\
Gel_H$_{2} \mathrm{SO}_{4}$ & 5.20 & 50.64 & $17.31(5.40)^{2}$ & - & 1.02 \\
Gel_HClSO$_{3}$ & 4.16 & 50.40 & $17.33(5.40)^{2}$ & $3.9^{4}(0.11)$ & 1.03 \\
\hline
\end{tabular}

${ }^{1}$ approximate formula weight $=\mathrm{C}_{2} \mathrm{H}_{3}-\mathrm{C}_{6} \mathrm{H}_{5} \cdot \mathrm{xSO}_{3} ;{ }^{2} \mathrm{mmol} \cdot \mathrm{g}{ }^{-1} ;{ }^{3}$ average number of sulfonic groups per phenyl ring; ${ }^{4}$ due to residual $\mathrm{SO}_{2} \mathrm{Cl}$ from incomplete basic hydrolysis.

The PEC of a gel-type styrenic resin cannot exceed $5.4 \mathrm{mmol} \mathrm{H}^{+} \mathrm{g}^{-1}$, corresponding to the functionalization of all the aromatic rings with one sulfonic group each. The simple inspection of PECs suggests that oleum gave an oversulfonated resin (Gel_oleum), and concentrated $\mathrm{H}_{2} \mathrm{SO}_{4}$ introduced more or less one sulfonic group per phenyl ring in $\mathrm{Gel}_{-} \mathrm{H}_{2} \mathrm{SO}_{4}$.

This simple analysis would also suggest that sulfonation was not quantitative in $\mathrm{Gel}_{-} \mathrm{HClSO}_{3}$, which seems rather surprising. Whereas sulfuric acid and oleum are neither soluble in the swelling 
agent (DCE) nor able to swell the starting resin, chlorosulfonic acid is miscible with DCE. On this basis, chlorosulfonic acid should access the swollen polymer framework easily in comparison with sulfuric acid or oleum. The difficulty of the latter two sulfonation agents to diffuse into the polymer framework is shown by the egg-shell distribution of sulfonic groups in partially sulfonic resins [11]. In these cases, the sulfonation starts from the external surface, then the first sulfonic (and hydrophilic) layer is swollen by $\mathrm{H}_{2} \mathrm{SO}_{4}$ (or oleum) which sulfonates a second layer just beneath the surface and so on. However, the comparison of PECs with the total sulfur and carbon from the elemental analysis gives a more detailed and appropriate insight on the effects of the different sulfonation methods.

Sulfur can be distributed among three different types of "sulfo"-groups: sulfonic $\left(-\mathrm{SO}_{2} \mathrm{OH}\right)$, sulfone bridges $\left(-\mathrm{SO}_{2}-\right)$ and chlorosulfonic $\left(-\mathrm{SO}_{2} \mathrm{Cl}\right)$. The latter were found, in little amount, only in $\mathrm{Gel}_{-} \mathrm{HClSO}_{3}$ as shown by the presence of chlorine in the material. To estimate the actual degree of sulfonation of the phenyl rings we assumed that the resins were essentially sulfonic polystyrene with a formula weight $\mathrm{C}_{2} \mathrm{H}_{3}-\mathrm{C}_{6} \mathrm{H}_{5} \cdot x \mathrm{SO}_{3}$ (we neglected both the presence of DVB, which is nominally only $2 \%$ of the resin and the different nature of the sulfo-groups), where $x$ is straightforward to be obtained from the ratio of the $C$ and $S$ weight percentages (Equation (1)):

$$
x=8 \times \frac{S(\% w t) \times A W(C)}{A W(S) \times C(\% w t)} \approx 3 \times \frac{S(\% w t)}{C(\% w t)}
$$

This number is also the degree of sulfonation, defined as the average number of "sulfo" groups per phenyl ring. The values of $x$ show that the starting gel-type resin is fully (at least) sulfonated in each case and confirm that a remarkable oversulfonation takes place with oleum. In line with previous reports of sulfonation of PS-DVB resins with oleum exceeding the limit of one sulfonic group per each phenyl ring of the polymer [18], the PEC of Gel_oleum is consistent with the presence of 1.17(5) sulfonic groups per phenyl ring i.e., ca. $17 \%$ of the phenyl rings bear two acidic groups. However, this does not entirely account for the total content of sulfur of Gel_oleum. It can be estimated that there are on average $0.15(5)$ sulfone bridges per phenyl ring, which amounts to ca. $11 \%$ of total sulfur in the resin. This also implies that ca. 30\% of the phenyl rings are involved in the formation of bridged pairs and if we assume that there are no triply sulfonated rings, almost half of the phenyl rings of Gel_oleum are doubly sulfonated in one form or the other.

The analytical data of Table 1 support an even more extensive formation of sulfone bridges in $\mathrm{Gel}_{2} \mathrm{HClSO}_{3}$. In this case oversulfonation does not take place and this mainly goes to detriment of the PEC, with ca. $77 \%$ of the phenyl rings bearing only one sulfonic group. As mentioned above, the presence of chlorosulfonic groups also gives a contribution to the difference between the total sulfur amount of $\mathrm{Gel}_{-} \mathrm{HClSO}_{3}$ and its PEC. However, it is relatively little $\left(0.11 \mathrm{mmol} \cdot \mathrm{g}^{-1}\right.$ out of 5.40 $\mathrm{mmol} \cdot \mathrm{g}^{-1}$ of total sulfur) and only ca. $2 \%$ of the phenyl rings bear $-\mathrm{SO}_{2} \mathrm{Cl}$ groups. The remaining ca. $21 \%$ of total sulfur of $\mathrm{Gel}_{-} \mathrm{HClSO}_{3}$ must be accounted for by the formation of sulfone bridges, which implies that more than $40 \%$ of them are involved in the formation of bridged pairs, but only about one fifth are doubly sulfonated. $\mathrm{HClSO}_{3}$ is therefore more apt to form sulfone bridges than oleum, which gives only $11 \%$ of total sulfur in this form, although it proves to be a weaker sulfonating agent in the conditions employed herein.

Finally, the analytical data of $\mathrm{Gel}_{-} \mathrm{H}_{2} \mathrm{SO}_{4}$ show that neither the oversulfonation nor the formation of sulfone bridges occur with sulfuric acid. Under the conditions employed in this work, the sulfonation leads readily to an essentially fully sulfonic resin.

All these results are completely in line with the well-known circumstance that the formation of sulfone bridges is a side reaction of the sulfonation with oleum and chlorosulfonic acid $[11,15,19,20]$. According to the literature [21], it involves the acid catalyzed condensation of two sulfonic group, in which the use of a very strong acid and dehydrating agent such as oleum is expected to favour both the protonation of the sulfonic groups and the subsequent elimination of water. Moreover, in doubly sulfonated rings, the acidity of the sulfonic groups is significantly enhanced [18], due to the deactivation of the aromatic unit, and the protonation step is expected to be even more effective. 


\subsection{Morphological Characterization in the Swollen State}

The swollen state morphology of cross-linked polymers can be conveniently investigated by means of ISEC $[7,22,23]$. This kind of materials can have specific surface areas and pore volumes much larger in the swollen state than in the dry state. Gel-type resins, for instance, are essentially non-porous and have specific surface areas of a few square-meters per gram only when dry. However, they can swell extensively in proper liquids. The intrusion of the molecules of the swelling agent into the polymer framework separates the polymer chains from one another. The framework expands and the space between the separated chains is filled by the molecules of the swelling agent. This process produces a gel in which the cavities, created by the separation of the chains, have characteristic size comparable to those of micropores and small mesopores. The specific gel volume and surface area can amount to a few cubic centimetres per gram and several hundreds of square meters per gram, respectively. Under these conditions, the interior of the polymer framework can be accessed from the liquid phase in contact with it and exploited for chemical purposes.

In this case, the resin morphology is best described with the Ogston model, which depicts the polymer chains as randomly oriented rigid cylindrical rods, with high aspect ratios, and the pores as void spaces among them [24]. In this model the characteristic quantity is the polymer chain concentration, the sum of the lengths of the rods which are contained in a unit volume of gel and which is generally expressed in $\mathrm{nm} \cdot \mathrm{nm}^{-3}$. Real swollen materials are generally described as a number of discrete gel fractions, each with its volume and characteristic polymer chain concentration. The result of ISEC analysis based on the Ogston model is therefore a volume distribution of different values of polymer chain concentration. As an alternative the cylindrical pore model could also be used, where the pores are depicted as cylinders with a specific pore diameter. This provides a much more direct estimation of the sizes of the cavities in the expanded polymer framework, although it is clear that nothing similar to pores in rigid materials can hardly exist in this case and that the Ogston model is closer to the real situation. The general relationship between the two models is that higher polymer chain concentrations correspond to smaller "pore diameters" and vice versa.

The ISEC characterization of $\mathrm{Gel}_{-} \mathrm{H}_{2} \mathrm{SO}_{4}$, Gel_oleum and $\mathrm{Gel} \_\mathrm{HClSO}_{3}$ provides important information on the effects of the sulfonation method on the nanometer scale morphology of the swollen gels. They are presented herein using the Ogston model, hence each material is divided in a set of six discrete domains with polymer chain concentrations ranging from 0.1 to $2 \mathrm{~nm} \mathrm{~nm}^{-3}$, which approximately correspond to pore diameters from 6 to $1 \mathrm{~nm}$. The results of ISEC characterization are summarized in Table 2 and illustrated in Figure 1.

Table 2. ISEC characterization of $\mathrm{Gel}_{-} \mathrm{H}_{2} \mathrm{SO}_{4}, \mathrm{Gel}$ _oleum, $\mathrm{Gel} \_\mathrm{HClSO}_{3}$.

\begin{tabular}{|c|c|c|c|}
\hline \multirow{2}{*}{$\begin{array}{l}\text { Polymer Chain Concentration } \\
\qquad\left(\mathrm{nm} \mathrm{nm}^{-3}\right)\end{array}$} & \multicolumn{3}{|c|}{ Polymer Chain Concentration $\left(\mathrm{nm} \mathrm{nm}^{-3}\right)$} \\
\hline & $\mathrm{Gel} \_\mathrm{H}_{2} \mathrm{SO}_{4}$ & Gel_oleum & $\mathrm{Gel}_{-} \mathrm{HClSO} \mathrm{O}_{3}$ \\
\hline 0.1 & 0.44 & 0.10 & 0.27 \\
\hline 0.2 & 1.30 & 0.68 & 0.37 \\
\hline 0.4 & 1.41 & 2.13 & 1.92 \\
\hline 0.8 & 0.00 & 0.00 & 0.00 \\
\hline 1.5 & 0.00 & 0.00 & 0.00 \\
\hline 2.0 & 0.00 & 0.00 & 0.00 \\
\hline Total gel volume $\left(\mathrm{mL} \mathrm{g}^{-1}\right)$ & 3.15 & 2.91 & 2.56 \\
\hline
\end{tabular}




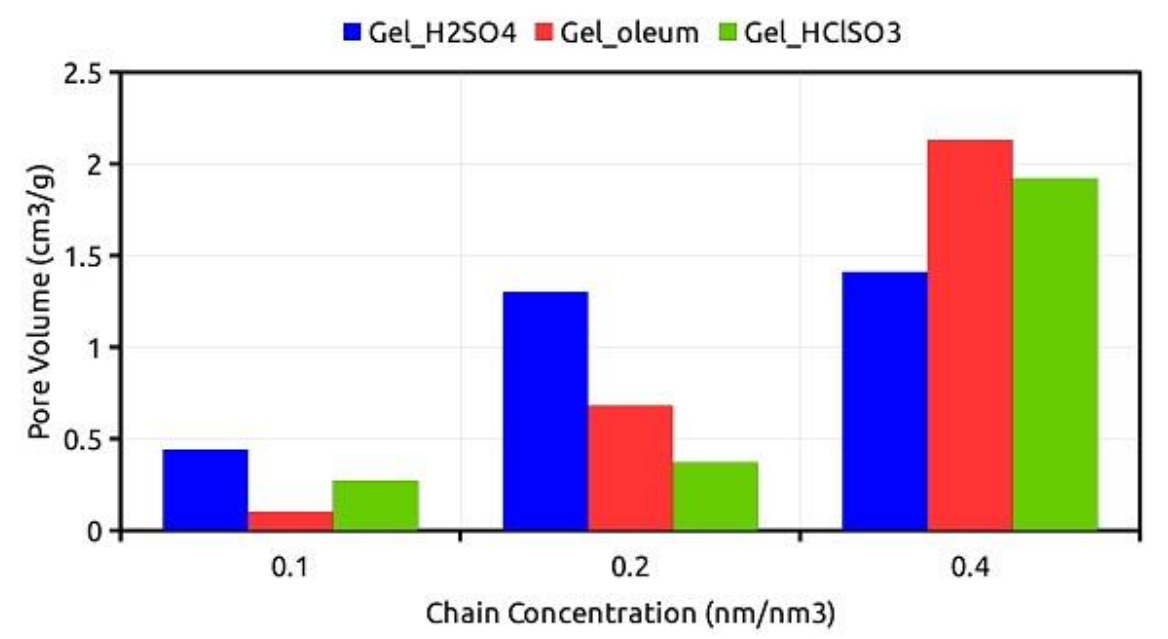

Figure 1. Distribution of the polymer chain concentration of $\mathrm{Gel}_{-} \mathrm{H}_{2} \mathrm{SO}_{4}, \mathrm{Gel}$ _oleum and $\mathrm{Gel} \_\mathrm{HClSO}_{3}$, according to ISEC.

Due to the hydrophilic nature of the sulfonic PS-DVB resins, an aqueous solution of sodium sulfate $(0.2 \mathrm{M})$ was used as the mobile phase in the ISEC runs. The presence of the electrolyte is necessary to suppress enthalpic contributions to the partition of the standard solutes between the stationary and mobile phases. The polymer chain concentration exceeds $0.4 \mathrm{~nm} \cdot \mathrm{nm}^{-3}$ and most of the polymer length (hence mass) is always found in the $0.4 \mathrm{~nm} \cdot \mathrm{nm}^{-3}$ and $0.2 \mathrm{~nm} \cdot \mathrm{nm}^{-3}$ fractions. However, whereas in $\mathrm{Gel}_{-} \mathrm{H}_{2} \mathrm{SO}_{4}$ these fractions have approximately the same volume (ca. $45 \%$ of the total gel volume), in Gel_oleum and $\mathrm{Gel}_{-} \mathrm{HClSO}_{3}$ the $0.4 \mathrm{~nm} \cdot \mathrm{nm}^{-3}$ has a much higher relative volume (ca. $75 \%$ of the total gel volume in both cases). This shows that Gel_oleum and $\mathrm{Gel}_{-} \mathrm{HClSO}_{3}$ have a lower swelling ability in water than $\mathrm{Gel}_{-} \mathrm{H}_{2} \mathrm{SO}_{4}$. Moreover, looking at the total gel volume Gel_oleum seems to swell slightly better than $\mathrm{Gel}_{-} \mathrm{HClSO}_{3}$. The relatively little swell-ability of $\mathrm{Gel}_{-} \mathrm{HClSO}_{3}$ could be simply attributed to its lower PEC $\left(4.16 \mathrm{mmol} \cdot \mathrm{g}^{-1}\right)$, which makes it slightly less hydrophilic than Gel_oleum and $\mathrm{Gel}_{-} \mathrm{H}_{2} \mathrm{SO}_{4}$ (PECs $=5.42$ and $5.20 \mathrm{mmol} \cdot \mathrm{g}^{-1}$, respectively). However, this argument cannot hold in the comparison of Gel_oleum and $\mathrm{Gel}_{-} \mathrm{H}_{2} \mathrm{SO}_{4}$. In this case, the reduced ability of Gel_oleum to swell could depend on the formation of sulfone bridges during the sulfonation. In fact, inter-chain bridges represent an additional form of cross-linking and the sulfur balance discussed in the previous section shows that this could amount up to $15 \%$. Such an additional cross-linking does not seem compatible with the relatively small difference in the swelling ability between Gel_oleum and $\mathrm{Gel}_{-} \mathrm{H}_{2} \mathrm{SO}_{4}$ and this suggests that most of the sulfone bridges could be of an intra-chain nature. Nevertheless, ISEC provides evidence that the formation of sulfone bridges is associated to the decrease in the swelling ability of sulfonic PS-DVB resins. The number of sulfone bridges estimated from the sulfur balance of $\mathrm{Gel}_{-} \mathrm{HClSO}_{3}$ in even higher and we cannot rule out that this could contribute to its relatively low swelling ability in addition to its lower PEC and hydrophilicity.

\subsection{ATR-FT-IR Characterization}

To support the relationship between the observed increased crosslinking degree and the presence of sulfone bridges, the sulfonic materials have been characterized with FT-IR spectroscopy to identify the characteristic signals of the expected functional groups. The spectra, collected in attenuated total reflectance (ATR) geometry, are reported in Figures $2-4$. 


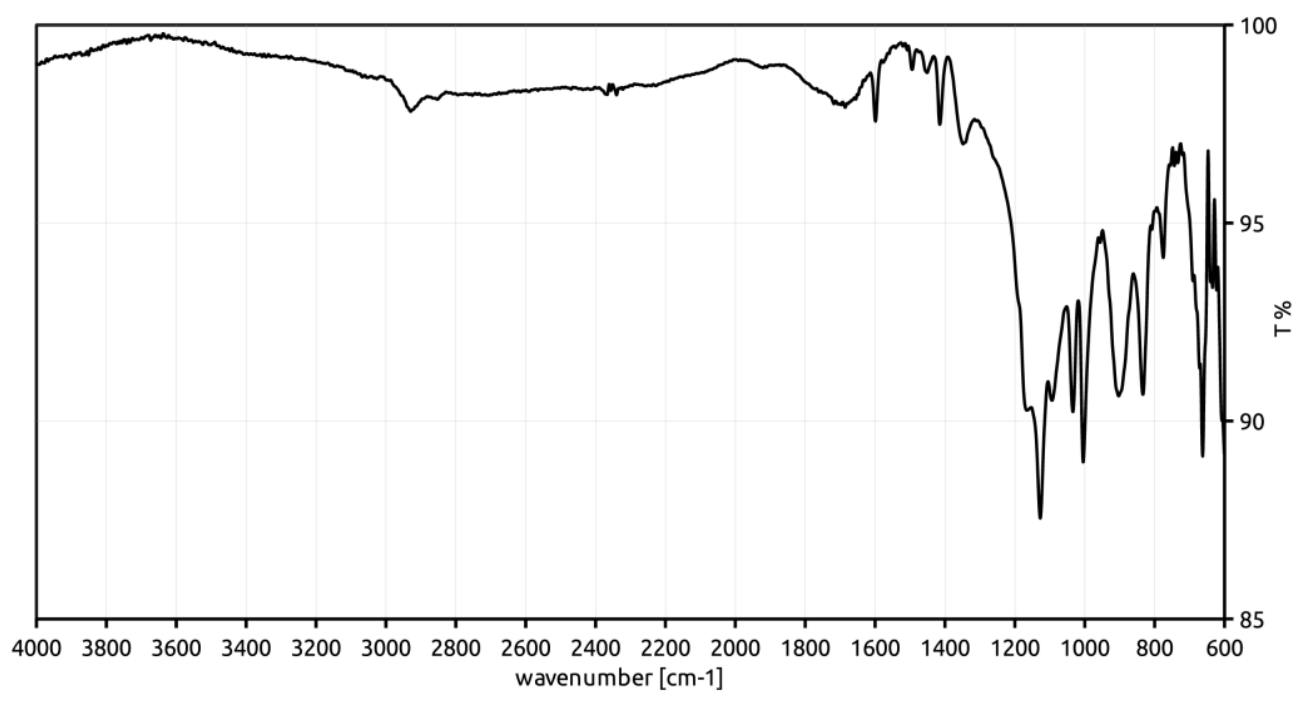

Figure 2. FT-IR spectrum of $\mathrm{Gel}_{-} \mathrm{H}_{2} \mathrm{SO}_{4}$.

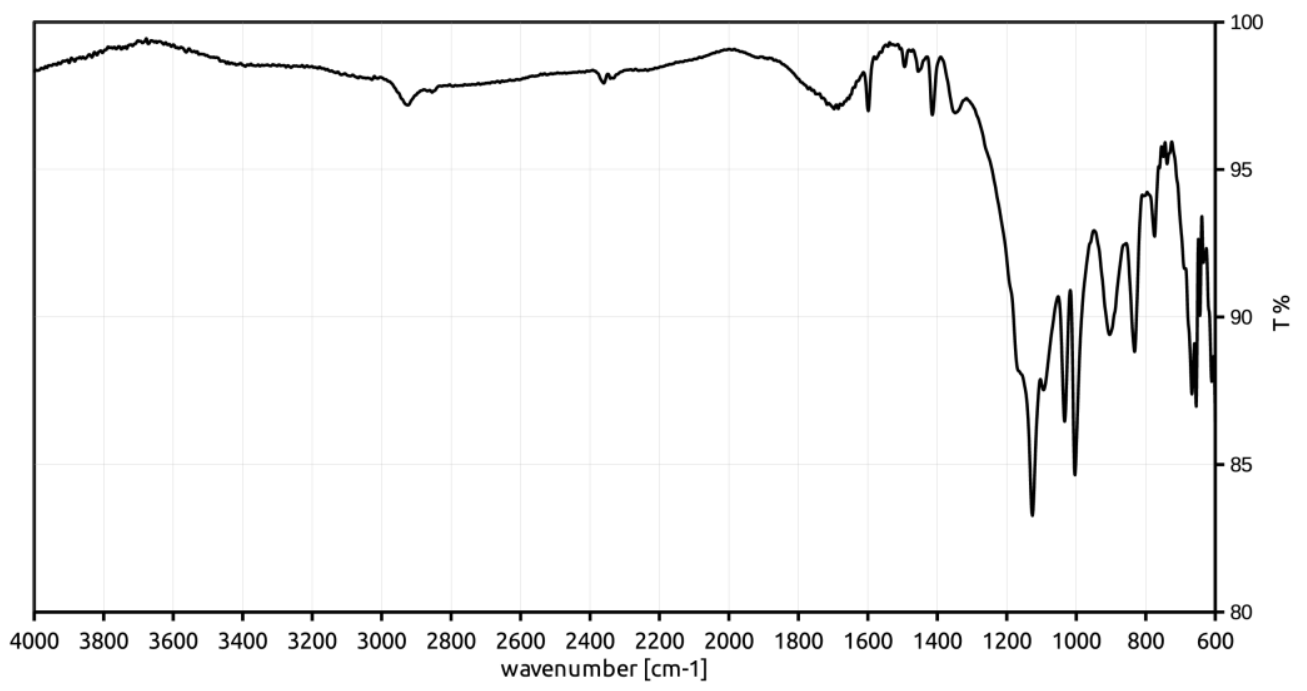

Figure 3. FT-IR spectrum of $\mathrm{Gel}_{-} \mathrm{HClSO}_{3}$.

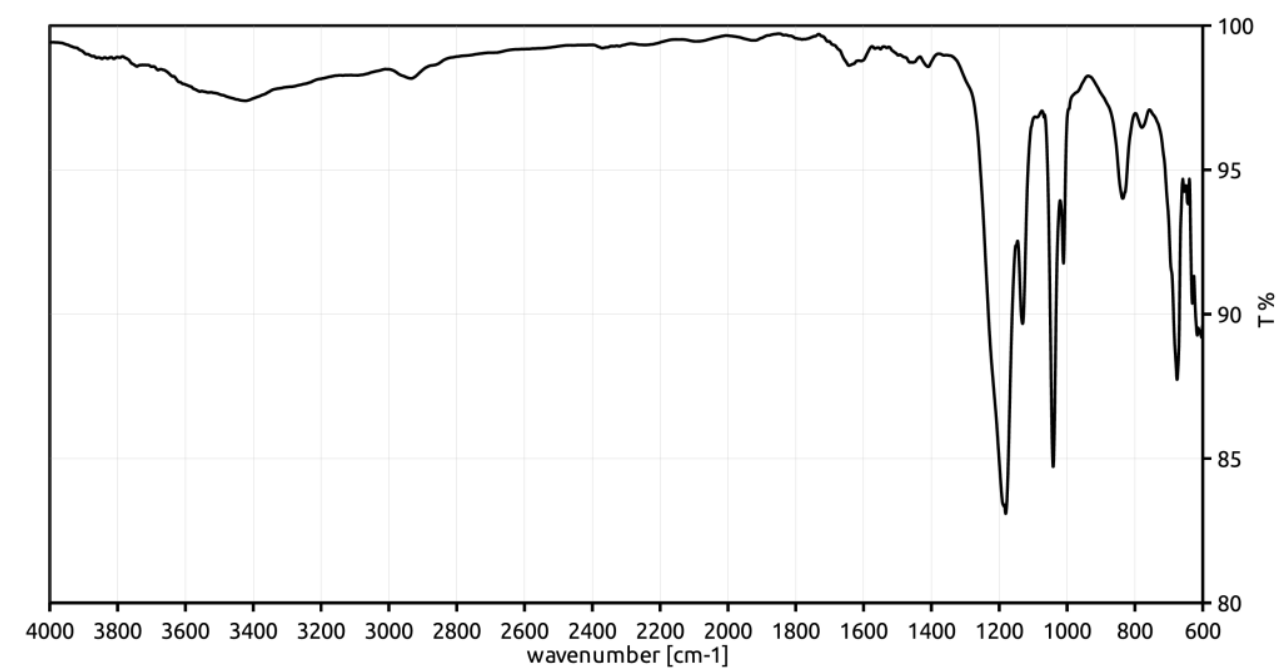

Figure 4. FT-IR spectrum of Gel_oleum. 
The relevant FT-IR data and the assignments of signals to vibrational modes are summarized in Table 3 [25].

Table 3. Diagnostic signals of sulfonic poly(styrene-divinylbenzene) materials, with the pertinent assignments.

\begin{tabular}{|c|c|}
\hline Peak/Band $\left(\mathrm{cm}^{-1}\right)$ & Assignment \\
\hline $1599,1495,1413$ & Skeleton stretching vibrations of the benzene ring \\
\hline 1451 & scissor vibration of $\mathrm{CH}_{2}$ group \\
\hline 1348 & antisymmetric stretching vibration of the $\mathrm{S}=\mathrm{O}$ bonds \\
\hline 1033 & symmetric stretching vibration of the $-\mathrm{SO}_{3}{ }^{-}$ion \\
\hline 1126,1097 & $\begin{array}{c}\text { in-plane skeleton vibration of the benzene ring. Bands characteristic of disubstituted } \\
\text { benzene rings }\end{array}$ \\
\hline 1004 & in-plane bending vibrations of the $\mathrm{CH}$ groups for benzene ring \\
\hline $836 / 831$ & $\begin{array}{l}\text { out-of-plane vibration of the pairs of } \mathrm{CH} \text { groups in the p-substituted benzene ring (in } \\
\text { comparison with p-toluensulfonic acid, } 817 \mathrm{~cm}^{-1} \text { ) }\end{array}$ \\
\hline 772 & $\begin{array}{l}\text { out-of-plane vibration of four } \mathrm{CH} \text { groups in the o-substituted benzene ring (as the } \\
\text { term of comparison, } 765 \mathrm{~cm}^{-1} \text { for o-toluensulfonic acid) }\end{array}$ \\
\hline
\end{tabular}

All the spectra contain peaks which can be attributed to the sulfonic groups, such as those at $1348 \mathrm{~cm}^{-1}$ and $1033 \mathrm{~cm}^{-1}$. The signals at 836 and $772 \mathrm{~cm}^{-1}$ suggest that the functionalization of the phenyl rings takes place mainly in the ortho- and para-positions with respect to the alkyl chains of the polymer framework. However, para- position appears the most likely, due to its relatively high distance from the sterically bulky alkyl chains of the polymer framework. Also, the signal at $907 \mathrm{~cm}^{-1}$ can be reasonably assigned to the stretching vibration of the $\mathrm{S}-\mathrm{O}$ bond of the $-\mathrm{SO}_{3} \mathrm{H}$ group, in spite of a significant shift from the literature $\left(897 \mathrm{~cm}^{-1}\right)$.

The signal at $1166 \mathrm{~cm}^{-1}$ (present as a shoulder in the spectra of Gel_oleum and Gel_HClSO 3 materials) has been considered by some authors as diagnostic for the sulfone bridges. In fact, the diphenyl sulfones show the symmetric stretching vibration of two S-O bonds between 1160 and $1164 \mathrm{~cm}^{-1}$ [26,27]. Conversely, a signal at $1172 \mathrm{~cm}^{-1}$, due to the symmetric stretching vibration of the $\mathrm{S}=\mathrm{O}$ bonds of the $\mathrm{SO}_{3} \mathrm{H}$ group, is also reported for sulfonic polystyrene materials [25]. Therefore, the signal at $1166 \mathrm{~cm}^{-1}$ cannot unambiguously support the presence of sulfone bridge in the sulfonic resins.

Moreover, the $\mathrm{SO}_{2}$-antisymmetric stretching of the $-\mathrm{SO}_{2} \mathrm{Cl}$ group is not recognized in the FT-IR characterization of $\mathrm{Gel}_{-} \mathrm{HClSO}_{3}$. Accordingly, the chlorine content is very low $\left(0.11 \mathrm{mmol} \mathrm{g}^{-1}\right)$ and the conversion of the sulfonyl chloride units into sulfonic groups in the second stage of sulfonation process can be considered almost complete.

To summarize, the FT-IR characterization of the sulfonic materials is compatible with the possible formation of sulfone bridges and suggest the para- position of the styrenic rings as the most probable position for the sulfonation.

\subsection{Solid State CP-MAS ${ }^{13} C-N M R$ Characterization}

A more detailed insight into the chemical nature of the sulfonic resins can be obtained with solid state CP-MAS NMR (Figures 5-7). For the sake of comparison, the NMR spectrum of the starting cross-linked polymer has also been collected (Figure 8). The occurrence of isotropic chemical shifts vs. spinning sidebands has been checked for, by comparing spectra registered at different MAS speeds. Deconvolution of the observed signals has been carried out taking into account the chemical non-equivalence of the different carbon atoms. 


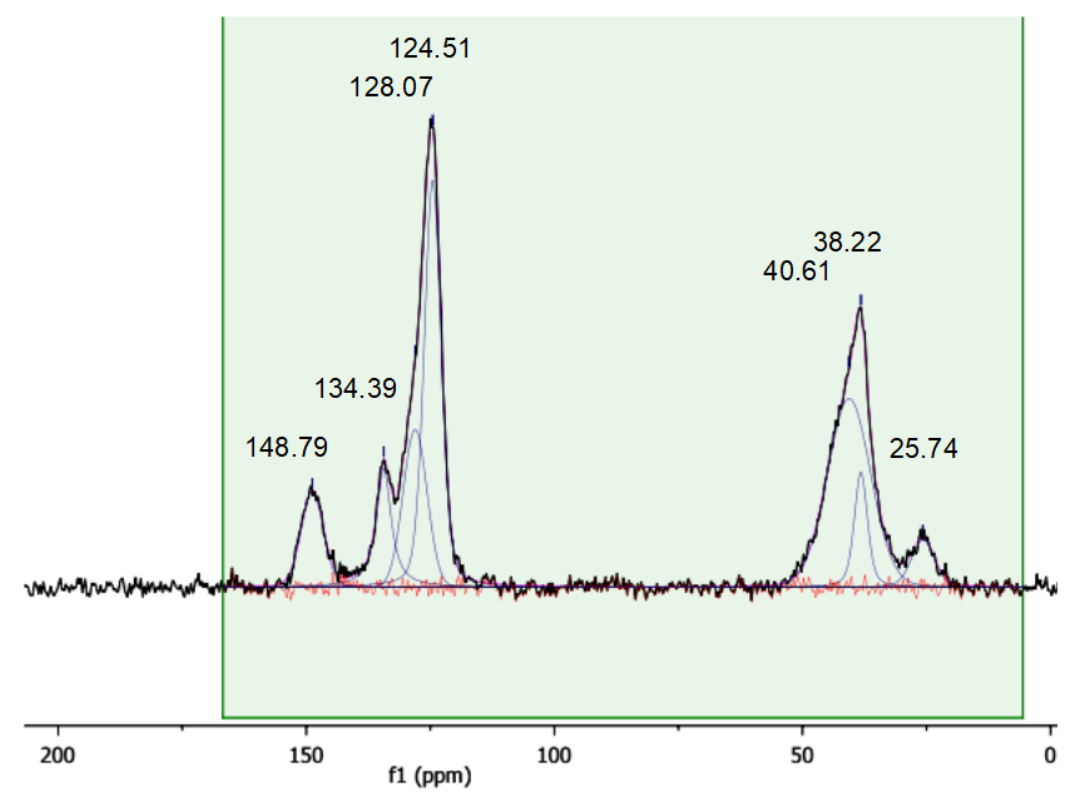

Figure 5. ${ }^{1} \mathrm{H}-{ }^{13} \mathrm{C} \mathrm{CP}$ MAS spectrum of $\mathrm{Gel}_{-} \mathrm{H}_{2} \mathrm{SO}_{4}$. Contact time $1 \mathrm{~ms}, \mathrm{MAS}$ rate $10 \mathrm{kHz}, \mathrm{T}=25{ }^{\circ} \mathrm{C}$.

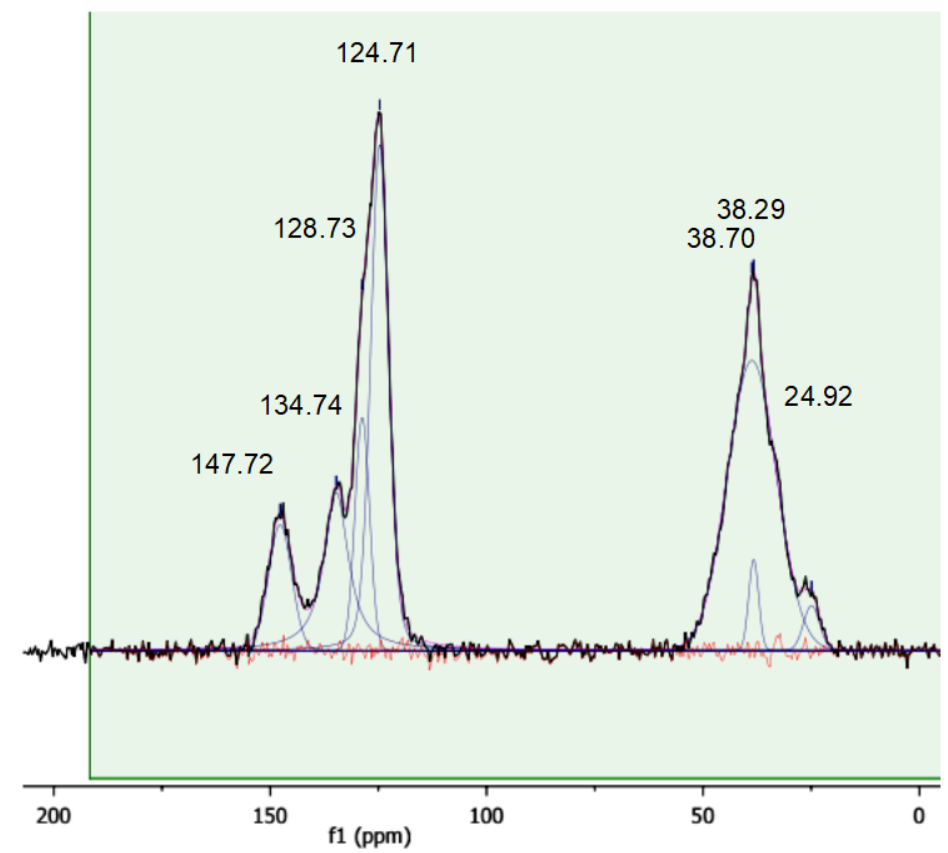

Figure 6. ${ }^{1} \mathrm{H}_{-}{ }^{13} \mathrm{C}$ MAS spectrum of Gel_oleum. Contact time $1 \mathrm{~ms}$, MAS rate $10 \mathrm{kHz}, \mathrm{T}=25{ }^{\circ} \mathrm{C}$. 


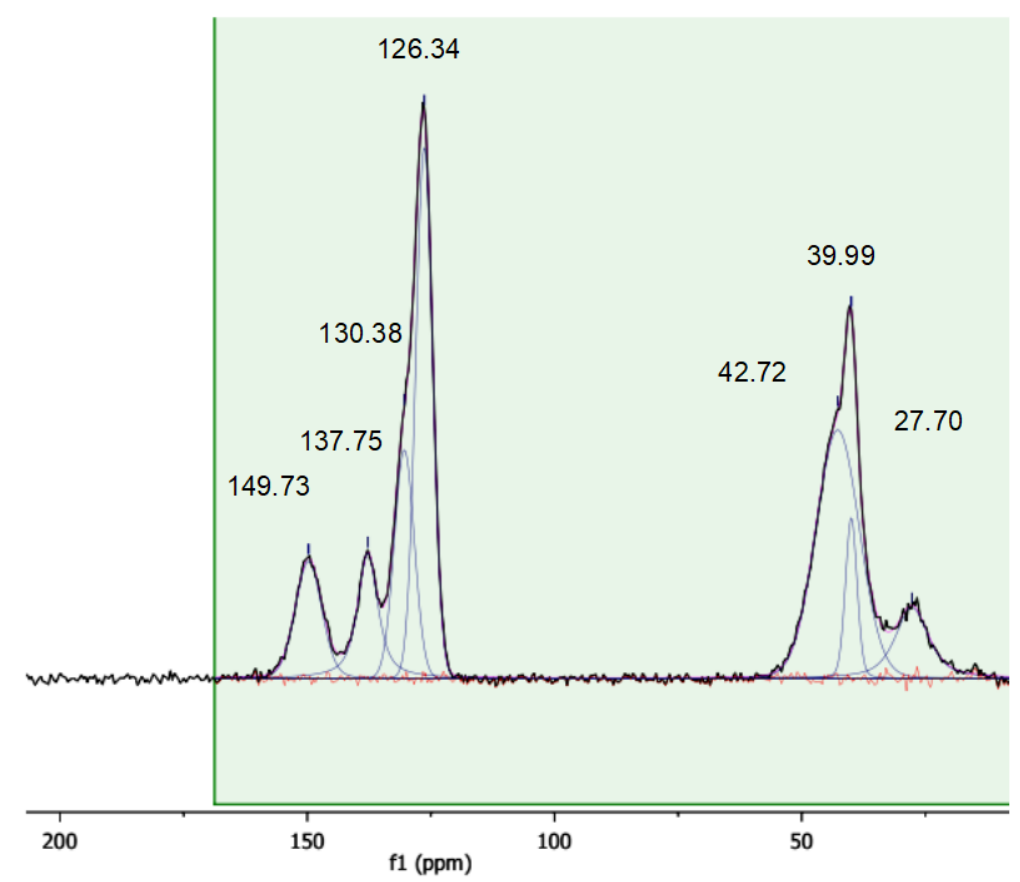

Figure 7. ${ }^{1} \mathrm{H}^{13} \mathrm{C}$ MAS spectrum of $\mathrm{Gel}_{-} \mathrm{HClSO}_{3}$. Contact time $1 \mathrm{~ms}$, MAS rate $10 \mathrm{kHz}, \mathrm{T}=25{ }^{\circ} \mathrm{C}$.

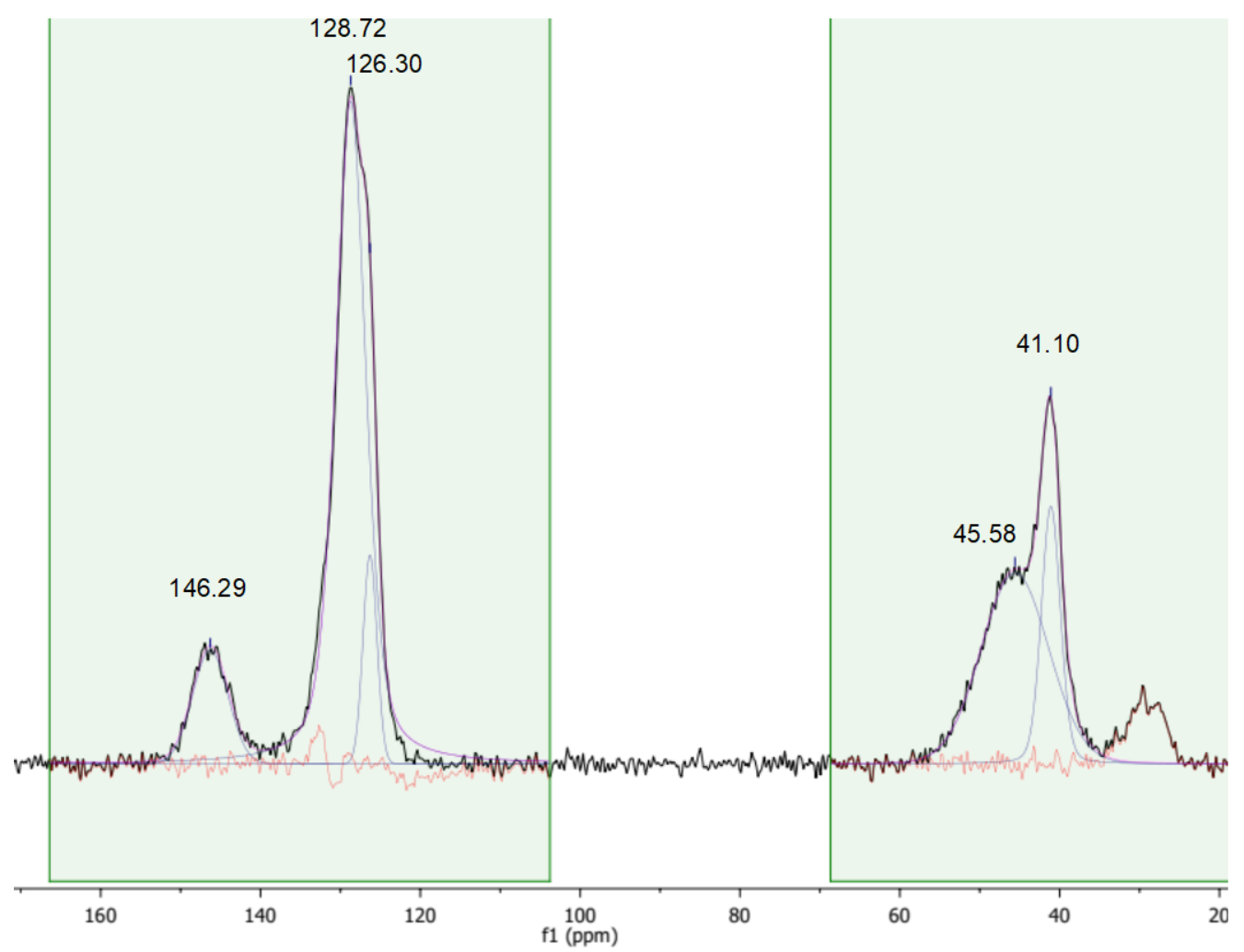

Figure $8 .{ }^{1} \mathrm{H}^{13} \mathrm{C}$ MAS spectrum of the starting gel-type poly(styrene-divinylbenzene) (2\% mol DVB). Contact time $1 \mathrm{~ms}$, MAS rate $=10 \mathrm{kHz}, \mathrm{T}=25^{\circ} \mathrm{C}$.

The signals at $41.10 \mathrm{ppm}$ and $45.58 \mathrm{ppm}$ in the spectrum of the starting material (Figure 8 ) can be assigned to the backbone carbons. The signal at $146.29 \mathrm{ppm}$ is due to the quaternary carbon of the aromatic rings, whereas the signals at 128.72 and $126.30 \mathrm{ppm}$ have been attributed to non-functionalized aromatic carbons [16]. 
The signals of sulfonic resins, summarized in Table 4, have been assigned according to the literature [28-30]. The positions of the carbon atoms of the aromatic rings given hereafter will be referred to the position of the polymeric chain.

Table 4. Signal attribution of ${ }^{1} \mathrm{H}^{13} \mathrm{C}$ MAS spectra of the sulfonic samples ( $\left.\delta, \mathrm{ppm}\right)$.

\begin{tabular}{ccccccc}
\hline & C $_{\text {ipso }}$ (1) & C $_{\text {para }}$ (4) & C $_{\text {ortho }}$ (2) & C $_{\text {meta }}$ (3) & \multicolumn{2}{c}{ C $_{\text {aliphatic }}$} \\
\hline Parent Gel resin & 146.3 & 126.3 & 128.7 & 128.7 & 45.6 & 41.1 \\
Gel_oleum & 147.7 & 134.7 & 128.7 & 124.7 & 38.7 & 38.3 \\
Gel_H $\mathrm{SO}_{4}$ & 148.8 & 134.4 & 128.1 & 124.5 & 40.6 & 38.2 \\
Gel_HClSO & 149.7 & 137.8 & 130.4 & 126.3 & 42.7 & 40.0 \\
\hline
\end{tabular}

The spectra of alkylbenzenes or 1,3-diphenylpropane and of p-toluensulfonic acid in $\mathrm{CDCl}_{3}$ [31] can be used to attribute the signals in the aromatic region by comparison. Accordingly, the aromatic carbon atom directly attached to the polymeric chain (ipso) resonates in the 146-150 ppm in all the materials. In the parent gel resin (non-sulfonic) the resonances of the ortho and meta carbon are superimposed at $128.7 \mathrm{ppm}$ (128.39 and $128.26 \mathrm{ppm}$ in 1,3-diphenylpropane) and the signal at $126.3 \mathrm{ppm}$ is assigned to the para- carbon atom. A signal in the $124.5-126.3 \mathrm{ppm}$ range is found also in the sulfonic polymers, but in this case, it can be attributed to the meta carbon atom, by analogy with p-toluensulfonic acid (125.45 ppm). The para position is the preferred one for the sulfonation of polystyrene [28-30,32] and the carbon atom bearing the sulfonic group resonates in the 134.4-137.8 ppm range. Not only is this signal absent in the spectrum of the parent gel resin, but its position is also consistent with the solution-state spectrum $\left(\mathrm{CDCl}_{3}\right)$ of p-toluensulfonic acid, where the sulfonated carbon atoms resonate at $138.49 \mathrm{ppm}$. Hence, in view of the degree of sulfonation obtained from the mass balance of sulfur, no unsubstituted para carbon atoms should be present hence.

As shown above in Gel_oleum, ca. 33\% of the aromatic rings are doubly sulfonated, but its spectral pattern is practically the same of the other two sulfonic resins. We therefore speculate that the sulfonated carbon atoms resonate at the same frequency irrespective of their position or that their resonances are so close that they cannot be resolved. The second sulfonation of the aromatic rings of Gel_oleum most likely occurs in the ortho- position, which is favoured by the orienting effects of both the (alkyl) polymeric chain and of the first "sulfo" group, assuming that the para position is sulfonated first. This is consistent with the well-know regiochemistry of aromatic electrophilic substitutions. In fact, the polymer chains can be considered as alkyl substituents of the phenyl rings at the ipso carbon atoms. Alkyls are electron-donating, para- and ortho-directing groups. As the polymeric chains are very bulky, the para- position should be preferred for the first sulfonation. In addition, the first sulfonic group introduced in the phenyl ring, which is electron withdrawing, is meta- directing (with respect to its own position). Thus, it should also contribute to the sulfonation of the ortho- position with respect to the ipso carbon atom. This is qualitatively confirmed by the analysis of the peak intensity in the spectra of the sulfonic resins (Table 5; for sake of clarity the carbon atoms are also numbered according to the structural scheme represented in Figure 9).

Table 5. Integrated intensities (absolute units) of the signals of aromatic carbon atoms.

\begin{tabular}{cccc}
\hline & Gel_H $_{2}$ SO $_{4}$ & Gel_oleum & Gel_HClSO \\
\hline $\mathrm{A}_{\text {ipso }}(1)$ & 723.785 & 1476.143 & 3467.159 \\
$\mathrm{~A}_{\text {sulfonated }}(2)$ & 834.452 & 2831.201 & 3574.537 \\
$\mathrm{~A}_{\text {ortho }}(3)$ & 1229.298 & 1716.835 & 4811.788 \\
$\mathrm{~A}_{\text {meta }}(4)$ & 2542.258 & 5038.13 & 9517.451 \\
$\mathrm{~A}_{\text {sulfonated }} / \mathrm{A}_{\text {ortho }}(4 / 1)$ & 0.68 & 1.66 & 0.75 \\
$\mathrm{~A}_{\text {meta }} / \mathrm{A}_{\text {ortho }}(3 / 1)$ & 2.1 & 2.87 & 1.99 \\
\hline
\end{tabular}


Gel_ $\mathrm{H}_{2} \mathrm{SO}_{4}$

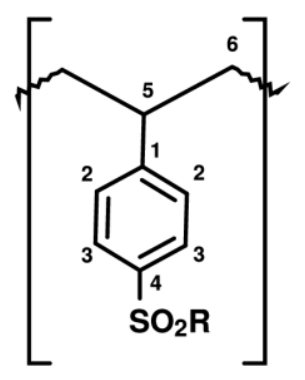

Gel_oleum

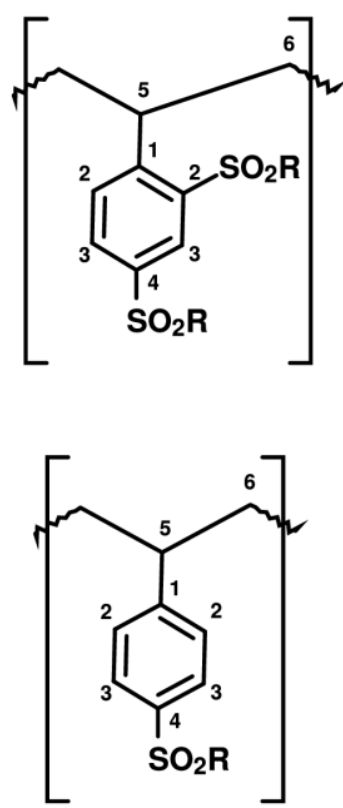

Gel_HClSO

Figure 9. Chemical structures of the samples, expected on the basis of comparison between elemental analysis and ion exchange capacities. The aromatic carbon atoms with similar chemical shifts are labeled with the same letters. The $\mathrm{R}$ group is either the $-\mathrm{OH}$ moiety (in the case of sulfonic group) or an aromatic ring (in the case of sulfone bridge).

Although the intensity of cross-polarized signals cannot be directly related to the number of resonating spins, the ratio between the areas of selected signals can be compared for similar samples. For $\mathrm{Gel}_{-} \mathrm{H}_{2} \mathrm{SO}_{4}$ and $\mathrm{Gel}_{-} \mathrm{HClSO}_{3}$, which have no doubly-sulfonated aromatic rings, the intensity ratios ortho/sulfonated and meta/sulfonated are similar. By contrast, for Gel_oleum the intensity ratios ortho/sulfonated and meta/sulfonated are much lower and much higher, respectively. This suggests, at least qualitatively, that the number of carbon atoms in ortho to the polymeric chain is smaller in Gel_oleum and supports the hypothesis of the second sulfonation of its aromatic rings in this position. A complete sulfonation in the ortho- position is anyway ruled out by the NMR data and also by the presence of the signal at $836 \mathrm{~cm}^{-1}$ in the IR spectra of the sulfonic resins.

\section{Conclusions}

The sulfonation of a $2 \%$ cross-linked, gel-type polystyrene-divinylbenzene resin with concentrated sulfuric acid, oleum and chlorosulfonic acid produces ion-exchange resins (Gel_oleum, Gel_ $\mathrm{H}_{2} \mathrm{SO}_{4}$, $\mathrm{Gel}_{-} \mathrm{HClSO}_{3}$ ) with different properties. The conditions employed allow to achieve full sulfonation of the resin and, with oleum even oversulfonation. Hence Gel_oleum has a PEC slightly higher than the ceiling value for a fully mono-sulfonic resin and, of course, higher than the PECs of $\mathrm{Gel}_{-} \mathrm{H}_{2} \mathrm{SO}_{4}$ and $\mathrm{Gel}_{-} \mathrm{HClSO}_{3}$. In view of its relatively high PEC, Gel_oleum is expected to be the most hydrophilic material hence to have the largest swelling ability in aqueous environment. In spite of this its swollen gel volume, as measured by ISEC, is somewhat lower than the swollen gel volume of $\mathrm{Gel}_{-} \mathrm{H}_{2} \mathrm{SO}_{4}$. The detailed morphological ISEC analysis also shows that in Gel_oleum ca. $73 \%$ of the gel volume is represented by the relatively most dense fraction $\left(0.4 \mathrm{~nm} \cdot \mathrm{nm}^{-3}\right)$, while it is only $44 \%$ in $\mathrm{Gel}_{-} \mathrm{H}_{2} \mathrm{SO}_{4}$. This counter-intuitive result can be attributed to the presence of sulfone bridges in Gel_oleum. From the mass balance of sulfur, it can be estimated that they connect ca. $30 \%$ of the aromatic rings of Gel_oleum while there are none in $\mathrm{Gel}_{-} \mathrm{H}_{2} \mathrm{SO}_{4}$. This implies that the actual cross-linking degree of Gel_oleum is higher (and hence its swelling ability is lower) than that of $\mathrm{Gel}_{-} \mathrm{H}_{2} \mathrm{SO}_{4}$. These conclusions are also supported by CP-MAS ${ }^{13} \mathrm{C}-\mathrm{NMR}$ spectroscopy. $\mathrm{Gel}_{-} \mathrm{HClSO}_{3}$ has the lowest swelling ability in aqueous 
environment and the highest proportion of sulfone bridges relative to both the number of aromatic rings and the total sulfur amount. However, it also has the lowest PEC (hence hydrophilicity), which is mostly the consequence of the aptitude of $\mathrm{HClSO}_{3}$ to give a high proportion of sulfone bridges and its inability to give oversulfonation (the contribution of the incomplete hydrolysis of the intermediate $-\mathrm{SO}_{2} \mathrm{Cl}$ groups to $-\mathrm{SO}_{3} \mathrm{H}$ is almost negligible). The reduced swelling ability of $\mathrm{Gel}_{-} \mathrm{HClSO}_{3}$ could therefore arise from one or both these causes. Further investigation is needed to find which one plays the most important role.

Supplementary Materials: The following are available online at http:/www.mdpi.com/2073-4360/12/3/600/s1.

Author Contributions: Conceptualization, P.C. and M.Z. methodology, P.C., M.Z. and F.R. investigation, C.D.V. and F.R. writing-original draft preparation, P.C. writing-review and editing, C.P., M.Z., F.R. and C.T. supervision, C.P. funding acquisition, P.C. and C.T. All authors have read and agreed to the published version of the manuscript.

Funding: This research was funded by University of Padova, PRAT project CPDA123471.

Acknowledgments: We are grateful to Karel Jeřábek for inspiring discussions on the swollen state morphology of ion-exchange materials.

Conflicts of Interest: The authors declare no conflict of interest. The funders had no role in the design of the study; in the collection, analyses, or interpretation of data; in the writing of the manuscript, or in the decision to publish the results.

\section{References}

1. Dorfner, K. Ion Exchangers; De Gruyter: Boston, MA, USA, 2011; ISBN 978-3-11-086243-0.

2. Sherrington, D.C.; Hodge, P. Syntheses and Separations Using Functional Polymers; Wiley: Hoboken, NJ, USA, 1988; ISBN 978-0-471-91848-6.

3. Jeřábek, K. Inverse Steric Exclusion Chromatography as a Tool for Morphology Characterization. In Strategies in Size Exclusion Chromatography; Potschka, M., Dubin, P.L., Eds.; American Chemical Society: Washington, DC, USA, 1996; Volume 635, pp. 211-224. ISBN 978-0-8412-3414-7.

4. Corain, B.; Zecca, M.; Canton, P.; Centomo, P. Synthesis and catalytic activity of metal nanoclusters inside functional resins: An endeavour lasting 15 years. Philos. Trans. R. Soc. Lond. Math. Phys. Eng. Sci. 2010, 368, 1495-1507. [CrossRef] [PubMed]

5. Zecca, M.; Centomo, P.; Corain, B. CHAPTER 10-Metal Nanoclusters Supported on Cross-Linked Functional Polymers: A Class of Emerging Metal Catalysts. In Metal Nanoclusters in Catalysis and Materials Science; Corain, B., Schmid, G., Toshima, N., Eds.; Elsevier: San Diego, CA, USA, 2008; pp. 201-232. ISBN 978-0-444-53057-8.

6. Jeřábek, K. Determination of pore volume distribution from size exclusion chromatography data. Anal. Chem. 1985, 57, 1595-1597. [CrossRef]

7. Jeřábek, K. Characterization of swollen polymer gels using size exclusion chromatography. Anal. Chem. 1985, 57, 1598-1602. [CrossRef]

8. Corain, B.; D’Archivio, A.A.; Galantini, L.; Lora, S.; Isse, A.A.; Maran, F. Electrochemical, Pulsed-Field-Gradient Spin-Echo NMR Spectroscopic, and ESR Spectroscopic Study of the Diffusivity of Molecular Probes inside Gel-Type Cross-Linked Polymers. Chem. Eur. J. 2007, 13, 2392-2401. [CrossRef] [PubMed]

9. Bortolus, M.; Centomo, P.; Zecca, M.; Sassi, A.; Jeřábek, K.; Maniero, A.L.; Corain, B. Characterisation of Solute Mobility in Hypercross-Linked Resins in Solvents of Different Polarity: Two Promising Supports for Catalysis. Chem. Eur. J. 2012, 18, 4706-4713. [CrossRef] [PubMed]

10. Centomo, P.; Jeřábek, K.; Canova, D.; Zoleo, A.; Maniero, A.L.; Sassi, A.; Canton, P.; Corain, B.; Zecca, M. Highly Hydrophilic Copolymers of N,N-Dimethylacrylamide, Acrylamido-2-methylpropanesulfonic acid, and Ethylenedimethacrylate: Nanoscale Morphology in the Swollen State and Use as Exotemplates for Synthesis of Nanostructured Ferric Oxide. Chem. Eur. J. 2012, 18, 6632-6643. [CrossRef]

11. Hanková, L.; Holub, L.; Jeřábek, K. Relation between functionalization degree and activity of strongly acidic polymer supported catalysts. React. Funct. Polym. 2006, 66, 592-598. [CrossRef]

12. Jeřábek, K.; Hanková, L.; Holub, L. Working-state morphologies of ion exchange catalysts and their influence on reaction kinetics. J. Mol. Catal. Chem. 2010, 333, 109-113. [CrossRef] 
13. Guilera, J.; Hanková, L.; Jeřábek, K.; Ramírez, E.; Tejero, J. Influence of the functionalization degree of acidic ion-exchange resins on ethyl octyl ether formation. React. Funct. Polym. 2014, 78, 14-22. [CrossRef]

14. De Dardel, F.; Arden, T.V. Ion Exchangers; Ullmann's Encyclopedia of Industrial Chemistry; Wiley-VCH Verlag GmbH \& Co. KGaA: Weinheim, Germany, 2012; Volume 19.

15. Goldstein, S.; Schmuckler, G. Sulfone formation during the sulfonation of crosslinked polystyrene. Ion. Exch. Membr. 1972, 1, 63-66.

16. Law, R.V.; Sherrington, D.C.; Snape, C.E.; Ando, I.; Korosu, H. Solid State ${ }^{13}$ C MAS NMR Studies of Anion Exchange Resins and Their Precursors. Ind. Eng. Chem. Res. 1995, 34, 2740-2749. [CrossRef]

17. Saalwächter, K.; Spiess, H.W. Solid-State NMR of Polymers. In Polymer Science: A Comprehensive Reference; Elsevier: San Diego, CA, USA, 2012; pp. 185-219. ISBN 978-0-08-087862-1.

18. Jeřábek, K.; Hanková, L.; Prokop, Z.; Lundquist, E.G. Relations between morphology and catalytic activity of ion exchanger catalysts for synthesis of bisphenol A. Appl. Catal. Gen. 2002, 232, 181-188. [CrossRef]

19. Theodoropoulos, A.G.; Tsakalos, V.T.; Valkanas, G.N. Sulfone-type crosslinks in sulfonation of macronet polystyrene backbone. Polymer 1993, 34, 3905-3910. [CrossRef]

20. Rabia, I.; Zerouk, J.E.; Mekhalif, S. Chlorosulfonyl styrene-divinylbenzene copolymer characteristics as a function of chlorosulfonation and chlorination reaction parameters. Polym. Adv. Technol. 1998, 9, 107-112. [CrossRef]

21. Freeman, D.H.; Goldstein, S.; Schmuckler, G. Homogeneous Sulfonation of Styrene-Divinylbenzene Copolymers with Oleum in Organic Solvents. Isr. J. Chem. 1969, 7, 741-749. [CrossRef]

22. Cerfontain, H. Mechanistic Aspects in Aromatic Sulfonation and Desulfonation; Interscience Publishers: New York, NY, USA, 1968.

23. Hart, M.; Fuller, G.; Brown, D.R.; Park, C.; Keane, M.A.; Dale, J.A.; Fougret, C.M.; Cockman, R.W. Acidities and catalytic activities of persulfonated poly(styrene-co-divinylbenzene) ion-exchange resins. Catal. Lett. 2001, 72, 135-139. [CrossRef]

24. Ogston, A.G. The spaces in a uniform random suspension of fibres. Trans. Faraday Soc. 1958, 54, $1754-1757$. [CrossRef]

25. Zundel, G. Hydration and Intermolecular Interaction: Infrared Investigations with Polyelectrolyte Membranes; Academic Press: New York, NY, USA, 2012.

26. Barnard, D.; Fabian, J.M.; Koch, H.P. Valence vibration frequencies and hydrogen bond formation of sulphoxide and sulphone groups. (Absorption spectra and structure of organic sulphur compounds. Part V.). J. Chem. Soc. Resumed 1949, 514, 2442-2454. [CrossRef]

27. Feairheller, W.R., Jr.; Katon, J.E. The vibrational spectra of sulfones. Spectrochim. Acta 1964, 20, 1099-1108. [CrossRef]

28. Cánovas, M.J.; Sobrados, I.; Sanz, J.; Acosta, J.L.; Linares, A. Proton mobility in hydrated sulfonated polystyrene: NMR and impedance studies. J. Membr. Sci. 2006, 280, 461-469. [CrossRef]

29. Law, R.V.; Sherrington, D.C.; Snape, C.E.; Ando, I.; Kurosu, H. Solid-State ${ }^{13}$ C MAS NMR Studies of Hyper-Cross-Linked Polystyrene Resins. Macromolecules 1996, 29, 6284-6293. [CrossRef]

30. Yang, J.C.; Jablonsky, M.J.; Mays, J.W. NMR and FT-IR studies of sulfonated styrene-based homopolymers and copolymers. Polymer 2002, 43, 5125-5132. [CrossRef]

31. AIST: Spectral Database for Organic Compounds, SDBS. Available online: http://sdbs.db.aist.go.jp/sdbs/cgibin/cre_index.cgi (accessed on 20 December 2019).

32. Su, Z.; Hsu, S.L.; Li, X. Spectroscopic and thermal studies of sulfonated syndiotactic polystyrene. Macromolecules 1994, 27, 287-291. [CrossRef]

(C) 2020 by the authors. Licensee MDPI, Basel, Switzerland. This article is an open access article distributed under the terms and conditions of the Creative Commons Attribution (CC BY) license (http://creativecommons.org/licenses/by/4.0/). 